\title{
PARADIPLOMACIA AMBIENTAL: LA COOPERACIÓN DESCENTRALIZADA HISPANO-BRASILEÑA
}

Fernando ReI

Profesor Titular de Derecho Ambiental de la Fundación Armando Alvares Penteado - FAAP. Profesor del Programa de Doctorado en Derecho Ambiental Internacional de la Universidad Católica de Santos. Director Científico de SBDIMA (Sociedad Brasileña de Derecho Internacional del Medio Ambiente). Contacto: fernandorei@ig.com.br. Rua São Carlos do Pinhal, 640, cj.71, 01333-000, São Paulo - SP, Brasil.

Valeria Cristina Farias

Abogada del Estado de São Paulo, Profesora de Derecho de la Universidade Paulista - UNIP y Escola Superior de Administração, Marketing e Comunicação - ESAMC. Contacto: vfarias@sp.gov.br. Rua Ivampa Lisboa, no. 27 apto. 71, 11055-120, Santos - SP, Brasil.

\section{Resumen}

Durante la primera década del siglo XXI, las actividades internacionales medioambientales de los gobiernos subnacionales han suscitado más y más el interés de representantes políticos y académicos, suponiendo uno de los temas de creciente investigación de la nueva gobernanza medioambiental global. Es que la necesidad de acciones prácticas para afrontar los problemas medioambientales globales, ha hecho posible y dado legitimidad a la progresiva aparición de nuevas formas de autoridad. Este artículo analiza el papel de los gobiernos de las Comunidades Autónomas (CCAA) españolas y de estados brasileńos en la gobernanza medioambiental global, particularmente en el régimen internacional de los cambios climáticos en el seno la Red de Gobiernos Regionales por el Desarrollo Sostenible (nrg4Sd).

\section{Palabras clave}

Paradiplomacia; Gobernanza medioambiental; Gobiernos subnacionales; Cooperación hispano-brasileña.

\section{Abstract}

During the early decade of the century, environmental international activities of subnational governments have attracted more and more interest from politicians and 
academics, supposing a topic of increasing research into new global environmental governance. Is that the need for practical action to address global environmental problems, have made possible and given legitimacy to the gradual appearance of new forms of authority. This article analyzes the role of the governments of the Spanish Autonomous Communities (CCAA) Spanish and Brazilian states in global environmental governance, especially in the international regime on climate change in the heart of the Network of Regional Governments for Sustainable Development (nrg4SD).

\section{Key words}

Paradiplomac; Environmental governance; Subnational governments; Spanish-Brazilian cooperation.

\section{Introducción}

En los últimos tres lustros, las actividades internacionales desarrolladas por los gobiernos subnacionales en el ámbito de una agenda paradiplomática ambiental global han sido objeto de creciente participación política e interés académico, especialmente en el régimen de los cambios climáticos.

Es indiscutible que las motivaciones de los gobiernos subnacionales para trabajar en la agenda ambiental global son múltiples, pero a pesar de la inexistencia de un motivo común, sí existe un reconocimiento generalizado respecto a que su implicación en los asuntos internacionales supone ya de por sí un cambio en el futuro de la diplomacia y en la conducción de esta agenda.

El objeto de este artículo es analizar en esta agenda ambiental global, la cooperación descentralizada entre algunos gobiernos subnacionales de las Comunidades Autónomas (CCAA) españolas y de estados federados de Brasil en el desarrollo del régimen internacional climático, en particular a partir de un análisis de la articulación de esos gobiernos subnacionales en la labor de la Red de Gobiernos Regionales por el Desarrollo Sostenible (nrg4SD).

A estos efectos, el artículo se estructura en cuatro secciones. La primera, presenta brevemente las características principales de la paradiplomacia mediante el análisis sobre su necesidad y el estudio del papel de los actores no estatales en la construcción de una necesaria gobernanza climática global en este escenario de transición.

La segunda sección describe la red nrg4SD y sus actividades como ejemplo práctico de paradiplomacia en el ámbito climático y sus retos. La tercera parte ofrece una visión de las actividades paradiplomáticas medioambientales, en el ámbito de una relación estratégicamente desarrollada por las CCAA y estados federados brasileños en el seno de la red nrg4SD. Por último, la cuarta sección presenta las conclusiones finales de este estudio. 


\section{Los Nuevos Actores en la Comunidad Internacional y la Paradiplomacia Medioambiental}

Las acciones de los gobiernos subnacionales para afrontar el cambio climático suponen uno de los aspectos de la nueva gobernanza medioambiental global. Dichas acciones se relacionaron inicialmente con temas internacionales económicos, considerando la globalización como gran paradigma (KEATING, 1999; HOCKING; 2004; ALDECOA, 2010; MILANI y RIBEIRO, 2011). No obstante, estas acciones se han desarrollado paulatinamente a otros ámbitos, permitiendo utilizar hoy en día el concepto de paradiplomacia como el creciente interés y participación directa de los gobiernos subnacionales en problemas medioambientales globales (BUENO DA SILVA, 2010).

En otras palabras, la paradiplomacia ambiental está considerada como la inclusión de las acciones de los gobiernos subnacionales en la dinámica del Derecho Ambiental Internacional, el cual viene reconociendo la participación progresiva de otros niveles de gobierno en la compleja agenda ambiental global.

El concepto de paradiplomacia se ha visto evolucionado en las dos últimas décadas debido a la dinámica de las agendas internacionales y a los cambios jurídico-institucionales nacionales acontecidos tanto en Estados europeos como latinoamericanos.

En literatura especializada se han identificado las distintas claves de este movimiento próspero más allá de las fronteras estatales. Es que las negociaciones para avanzar en el régimen internacional de la lucha contra el calentamiento global están actualmente limitadas por la resistencia de los Estados para asumir nuevos y mayores compromisos (BERSTEIN et al, 2010), aunque estemos en las puertas de la Conferencia de las Partes de Paris.

Esta resistencia sigue socavando la legitimidad de los Estados como interlocutores privilegiados para la solución de la problemática del cambio climático al tiempo que refuerza a otras autoridades y centros de toma de decisión, concretamente a los gobiernos subnacionales. En verdad los actores subnacionales se ven forzados a ofrecer una respuesta más efectiva y proactiva que los Estados porque las emisiones de gases de efecto invernadero - GEI y los impactos del cambio climático se perciben y padecen en los niveles local y regional (SETZER, 2013). Por ello, a pesar de las restricciones constitucionales a las que se enfrentan algunos gobiernos subnacionales para desarrollar lo que se conoce como relaciones exteriores ${ }^{1}$, en la práctica su presencia en foros internacionales es cada vez más

1 Véase el caso de Brasil, donde el Ministerio de Relaciones Exteriores, tradicionalmente muy celoso de la conducción de la política externa del país, amparado en el monopolio jurídico-constitucional de la Unión en las relaciones exteriores, pasa a reconocer a partir de los años 90 del siglo pasado la progresiva intensidad de flujos internacionales de gobiernos subnacionales. La formalización de este reconocimiento se da bajo la creación, en 1997, de un órgano llamado Asesoría de Relaciones Federativas (ARF), vinculado directamente al Gabinete de la Cancillería. 
activa. Así pues, al hablar de paradiplomacia ambiental, se hace referencia a las condiciones necesarias para iniciar un proceso de vinculación internacional en una agenda donde la mayoría de los gobiernos subnacionales tienen competencias legislativas y administrativas sobre las fuentes más importantes de emisión de GEI (REI et al, 2012).

De esta manera, el alcance descentralizado de las Convenciones Multilaterales sobre temas ambientales ha llevado a muchos gobiernos subnacionales a albergar el deseo de participación en esos regímenes, siendo que el avance de los efectos regionales del cambio climático ha reforzado el carácter universal, y al mismo tiempo de gestión descentralizada, de este fenómeno. No en vano, muchas de las formas de producción y consumo consideradas como causa antrópica del cambio climático continúan existiendo y extendiéndose por todo el planeta causando graves impactos en todos los niveles (local, regional y global) y ámbitos (medioambiental, social, económica y política) de la comunidad (MATEO, 1992). Por esta razón, la lucha contra los problemas medioambientales globales se ha convertido en un reto para las actividades diplomáticas y una oportunidad para nuevas arquitecturas jurídicas.

En la política global, y concretamente en el régimen climático actual, asistimos a una tendencia de apatía de la autoridad nacional acompańada de una apertura hacia un nuevo sistema global que exige nuevas modalidades para luchar contra el cambio climático. La gobernanza medioambiental global sigue esta dirección (LUNA PONT, 2011).

Al fin y al cabo la dualidad global-local del cambio climático exige responsabilidad en todos los niveles de la organización social para afrontar este fenómeno (LITFIN, 2000). Esta nueva concepción de la responsabilidad supone que el mundo es más interdependiente que antes, lo que confirma una nueva lógica del poder en las relaciones internacionales (BULKELEY, 2010). En otras palabras, los problemas globales medioambientales, entre otros, tendrán soluciones satisfactorias únicamente si son negociados y regulados por todos los Estados, y si en esas negociaciones y regulaciones se tiene en cuenta la creciente importancia de los nuevos agentes en el escenario internacional.

La complejidad de la respuesta internacional así como la necesidad de acciones prácticas para afrontar los problemas medioambientales globales, han hecho posible y dado legitimidad a la progresiva aparición de nuevas formas de autoridad, las cuales han reclamado una revisión de la lógica y de la arquitectura del régimen internacional del cambio climático.

$\mathrm{Al}$ margen de las controversias que todavía puedan existir sobre el gravedad del cambio climático, sí existe un consenso sobre las mudanzas que este fenómeno comporta en el modelo tradicional estatocéntrico, el cual se orienta cada vez más hacia un nuevo sistema de relaciones complejas y en múltiples niveles (LUNA PONT, 2011). A pesar de carecer de soberanía, elementos de autonomía o control, la sociedad ha concedido a estas nuevas 
estructuras una legitimidad voluntaria (DEDUERWAERDERE, 2005). El motivo para ello es la consideración de que para afrontar de manera efectiva los problemas medioambientales globales es necesaria la acción coordinada y de cooperación entre los sistemas de gobierno de todos los niveles - estatal, supraestatal, infraestatal, interestatal -, destacando sobre todo la participación del nivel estatal, infraestatal (regional y local) y de los actores no gubernamentales, con la consideración de que todos estos niveles juegan una variedad de papeles (BULKELEY, ${ }^{2005)}$. Así es como comienza a existir una nueva gobernanza medioambiental global.

Es cierto que las iniciativas de los gobiernos subnacionales para afrontar el cambio climático han ampliado el alcance de los resultados de la gobernanza ambiental (RABE, 2004; ROSENZWEIG et al., 2010; BULKELEY e NEWELL, 2010). En algunos casos, estas actividades se suman a los esfuerzos nacionales e internacionales para afrontar el problema, ayudando a los Estados a cumplir sus compromisos internacionales (OKERE$\mathrm{KE}$ et al., 2009). En otros casos, las acciones subnacionales (domésticas) que producen una reducción efectiva de gases de efecto invernadero (GEI) pueden suponer una presión - directa o indirecta - sobre los propios Estados (GRAZIANO y REI, 2009) para avanzar en sus compromisos y esfuerzos en las negociaciones internacionales sobre el cambio climático.

Es decir, a través de la gobernanza medioambiental global se han creado distintas formas y niveles complementarios de lucha contra los problemas medioambientales. Como consecuencia de ello, el avance en los regímenes consistentes en la firma de tratados multilaterales se ha sustentado en actividades desarrolladas en los niveles infraestatal y transnacional por actores que no son parte formal en el sistema legal internacional. Además, existe una dinámica de influencia mutua entre el régimen y la gobernanza de la que se nutre el Derecho Ambiental Internacional.

Otro factor a tener en cuenta es el reconocimiento mutuo entre los gobiernos subnacionales de sus respectivas iniciativas jurídicas y políticas tanto a nivel internacional como transnacional para luchar contra los problemas ambientales globales. En estas iniciativas se incluyen actividades como la firma de acuerdos y alianzas entre gobiernos subnacionales de distintos países, la participación en conferencias y eventos internacionales, así como la participación en redes internacionales y transnacionales de gobiernos subnacionales (REI et al.,2012).

Efectivamente, y pesar de las limitaciones propias por la carencia de la condición de sujeto internacional, en la práctica los gobiernos subnacionales crean entre sí relaciones técnicas con vínculos jurídicos, y participan de forma original y en coalición en los regímenes jurídicos y organismos internacionales que regulan los compromisos de los Estados. Esta práctica ha influido en la naturaleza de las relaciones internacionales, basada tradicionalmente en la cooperación entre Estados, y ha introducido otros niveles 
de cooperación, apareciendo así las dimensiones local-regional-global. En consecuencia, las relaciones internacionales ofrecen en la actualidad tal grado de complejidad, que es necesario un gran cuidado y sensibilidad en su práctica, con objeto de no fomentar conflictos de competencias entre los diferentes niveles de gobierno respecto a sus vínculos internacionales.

Tal influencia resulta verdaderamente decisiva cuando los gobiernos subnacionales actúan en cooperaciones horizontales y redes solidarias (SETZER, 2013).

Así pues, el estudio de una red supone una importante herramienta para analizar y comprender la dinámica de las relaciones entre espacios y actores, las cuales se despliegan en ciertas áreas como el desarrollo sostenible, y en particular en el cambio climático. La función de una red es ejercer una conexión para fomentar las relaciones entre los actores y las estructuras técnicas de acuerdo a determinados objetivos específicos, que en algunos casos, pueden ser también políticos. Por tanto, las redes van adquiriendo un papel cada vez más reconocido, y van asumiendo progresivamente un importante espacio en los procesos multilaterales de decisión, lo cual supone cambios en la estructura organizativa y las relaciones de gobernanza.

Este es el caso de la Red de Gobiernos Regionales por el Desarrollo Sostenible (nrg4SD).

\section{La Red de Gobiernos Regionales por el Desarrollo Sostenible - nrg4SD}

Nrg4SD es una coalición internacional que reúne a nivel mundial a gobiernos subnacionales y asociaciones regionales de gobiernos subnacionales. La red promueve el entendimiento, la colaboración y las asociaciones en materia de desarrollo sostenible, y persigue un mayor reconocimiento internacional de la importancia de las contribuciones de los gobiernos subnacionales (únicamente regionales) al desarrollo sostenible.

La red se creó en una Conferencia paralela celebrada por 23 gobiernos subnacionales $^{2}$ y 4 asociaciones ${ }^{3}$ regionales de gobiernos subnacionales en el marco de la Cumbre

2 Firmantes de La Declaración de Gauteng: Australian Capital Territory, Australia; País Vasco, España; Baviera, Alemania; Cataluña, Espańa; Provincia de Entre Ríos, Argentina; Flandes, Bélgica; Gauteng, Sudáfrica; Goiás, Brasil; Greater London Authority, Inglaterra; Lapland, Finlandia; Mato Grosso, Brasil; Mpumalanga, Sudáfrica; Pennsylvania, EEUU; Pernambuco, Brasil; Poitou - Charentes, Francia ; Rio de Janeiro, Brasil ; Rio Grande do Sul, Brasil; Tocantins, Brasil; Toscana, Italia; Wallonia, Bélgica; Gales, Reino Unido; West Java, Indonesia; Western Australia, Australia.

www.nrg4sd.org/sites/default/files/default/files/content/public/11-elibrary/corporate/gauteng-es.pdf. Aceso en 2 de abril de 2015.

3 Association of European Regions; Conference des Regions Peripheriques Maritimes; The Northern Forum y Committee of the Regions of the European Union. www.nrg4sd.org/sites/default/files/default/ files/content/public/11-elibrary/corporate/gauteng-es.pdf. Aceso en 2 de abril de 2015. 
Mundial sobre Desarrollo Sostenible de Johannesburgo (2002). Como fruto de esta Conferencia paralela, se adoptó la Declaración de Gauteng, la cual no solo pone de manifiesto la importancia de los gobiernos subnacionales en los asuntos de desarrollo sostenible y la utilidad de la cooperación en el nivel subnacional, sino que también recoge el deseo de crear una red de gobiernos subnacionales de ámbito universal para el desarrollo sostenible (DECLARACIÓN DE GAUTENG, 2002). En el año siguiente, se celebra la Conferencia de San Sebastián de 2003, con la participación de 55 delegaciones, en la cual se propone el objetivo de lograr personalidad jurídica para la red. Ésta se logra un año más tarde, momento en el que la red es registrada como una "Asociación Internacional sin ánimo de lucro" al amparo de la legislación belga.

Nrg4SD trabaja para defender la labor de los gobiernos subnacionales en el desarrollo sostenible, particularmente en la acción climática. Durante sus casi 15 años de vida ha doblado su número de miembros, registrando en la actualidad más de 50 miembros de derecho de 30 países y 7 asociaciones de regiones, además de observadores, en los cinco continentes. Asimismo, nrg4SD representa a más de 1000 gobiernos subnacionales a nivel mundial, ya que en virtud de su calidad de miembro del Foro ${ }^{4}$ Global de Asociaciones de Regiones (FOGAR), ha adquirido la responsabilidad de representar a este Foro en los asuntos relativos al desarrollo sostenible.

Para coordinar sus actividades, nrg4SD ha creado un Steering Committee (Comité Directivo), que actúa como órgano de gobierno de la red. El Steering Committee se reúne dos veces al año en sesiones ordinarias, sin perjuicio de lo cual, pueden celebrarse reuniones extraordinarias. Este órgano persigue constantemente lograr un equilibrio geográfico en la red, para lo cual promueve la condición de miembro entre gobiernos subnacionales de países en desarrollo. Los miembros del Steering Committee se eligen por un periodo de tres años sin restricciones para la reelección, éstos eligen a su vez de entre sus miembros dos Presidentes, cinco Puntos Focales continentales y una Tesorería.

El Steering Commitee tiene las siguientes funciones: guiar y supervisar el trabajo de la Secretaría General y de la Tesorería; solicitar a la Secretaría General y a la Tesorería la producción de informes regulares sobre la implementación del Programa de Trabajo y los asuntos financieros de la red; supervisar la preparación del informe anual y cuentas anuales para ser sometidos a la Asamblea General'; y convocar las reuniones anuales de la Asamblea General (REI et al., 2013b).

4 FOGAR ha sido creado en Ciudad del Cabo, Sudáfrica, en agosto de 2007, tomando como base la "declaración de las regiones sobre su participación en la gobernanza de la globalización" firmada en Marsella, Francia, con motivo de la primera convención internacional a favor de un enfoque territorial del desarrollo. Con sus diecisiete redes de regiones fundadoras procedentes de todos los continentes, el FOGAR reúne a más de 1000 Regiones. Conforme www.regionsunies-fogar.org/. Aceso en 2 de abril de 2015.

5 nrg4SD. Network of Regional Governments for Sustainable Development. Available at http://www. nrg4sd.org/. Aceso em 2 de abril de 2015. 
Con objeto muy particular de defender la importancia del papel y de las acciones de los gobiernos subnacionales contra el calentamiento global, nrg4SD ha sido acreditada oficialmente ante la UNFCCC como observador social. Además, nrg4SD cuenta con un Grupo de Trabajo permanente que sigue las negociaciones internacionales sobre cambio climático y ha lanzado recientemente un Grupo de Trabajo Ejecutivo para la financiación de la acción climática a nivel subnacional. Todo esto ha permitido a lo largo de estos años a nrg4SD trabajar directamente con el Secretariado de la Convención y con algunas Partes de la misma, así como representar directamente en las negociaciones internacionales a los gobiernos subnacionales miembros de la red. En estas negociaciones, nrg4SD (i) defiende de forma constante un mayor reconocimiento de la crucial aportación de los gobiernos subnacionales a la acción sobre el cambio climático, (ii) trabaja con el Secretariado y los grupos de trabajo de la UNFCCC en temas técnicos y (iii) aporta de forma regular la presencia e iniciativas (NRG4SD, 2011) de las delegaciones subnacionales a los debates.

Con objeto de influir en el proceso de negociación sobre cambio climático, la red ha venido trabajando de forma individual con otras redes de gobiernos subnacionales, como The Climate Group (TCG), los Gobiernos Locales para la Sostenibilidad (ICLEI) y las Ciudades y Gobiernos Locales Unidos (UCLG). Esta colaboración entre redes de gobiernos subnacionales supone un esfuerzo para transformar la creciente concienciación ciudadana en una acción cotidiana respecto al cambio climático. También representa un contexto sin precedentes de los gobiernos subnacionales para contribuir a la adaptación de la gobernanza internacional del cambio climático a los tiempos modernos (REI et al., 2013b).

La red ha sido capaz de organizar diversas reuniones de envergadura sobre cambio climático, las cuales han puesto de manifiesto que las experiencias de los gobiernos subnacionales son la base de las políticas sobre cambio climático. En este sentido, en el camino hacia Río +20, que ocurrió en junio de 2012, nrg4SD ha participado muy activamente en las principales reuniones internacionales, a partir de un Grupo de Trabajo establecido para coordinar las obras de la red en este campo, y estuvo totalmente involucrada en el seguimiento del proceso de negociación del "Draft Zero", con el soporte estratégico de dos de sus miembros, los gobiernos subnacionales brasileños de Rio de Janeiro y São Paulo.

Cabe aún destacar el evento paralelo organizado por la nrg4SD en la última COP, em Lima, donde se ha dado seguimiento a la hoja ${ }^{6}$ de ruta de cambio climático establecida en la COP de Varsóvia, que propone a nrg4SD y sus miembros una dinámica general de trabajo y actividades concretas para desarrollar mensajes comunes y documentos estratégicos de apoyo dirigidos al proceso internacional, concretamente: la COP 21 de París.

6 Disponible en http://www.nrg4sd.org/sites/default/files/content/public/cc_roadmap_2014-2015.pdf 
Fruto de este esfuerzo institucional de nrg4SD, los gobiernos subnacionales han sido reconocidos, por primera vez, en un acuerdo de la UNFCCC. Concretamente, la sección titulada "La visión compartida para la Acción Cooperativa a Largo Plazo" de los Acuerdos de Cancún (Decisión 1/CP16), reconoce formalmente "la necesidad de comprometer a una amplia gama de stakeholders de los niveles global, regional, nacional y local, en las reducciones de emisiones legalmente obligatorias", y añade que "mientras existan estas implicaciones, el Protocolo tendrá un futuro efectivo. Se trata de una demanda clave respecto a los países en desarrollo". Además, en estos acuerdos se recoge el papel de los gobiernos subnacionales en los esfuerzos de adaptación, reducción de las emisiones en bosques y cooperación al desarrollo (UNFCCC, 2011).

Cabe añadir que nrg4SD también ha alcanzado logros en el marco de otras Convenciones de Naciones Unidas, concretamente las relativas a la biodiversidad. Al respecto, gracias a la destacada participación de los gobiernos regionales de Cataluña, Québec y São Paulo, y a la importante labor de coordinación realizada por la Secretaría de la red, se logró la adopción de la Decisión X/22 por las Partes en la reunión de la Convención de Naciones Unidas para la Biodiversidad Biológica (UNCBD) celebrada en octubre de 2010 en Nagoya. La Decisión X/22 recoge el Plan de Acción para los Gobiernos Subnacionales, Ciudades y Otras Autoridades Locales, y en ella, por un lado, se invita a las Partes a involucrar a los gobiernos subnacionales, ciudades y otras autoridades locales en la revisión de las estrategias y planes de acción nacionales relativos a la biodiversidad, y por otro lado, se insta a los gobiernos subnacionales, ciudades, autoridades locales y a sus redes a contribuir a la puesta en funcionamiento del Plan de Acción. Asimismo, el Plan de Acción posibilita la creación de Comités Consultivos de Ciudades y Gobiernos Subnacionales bajo la figura de asociaciones y mecanismos de coordinación (REI et al., 2013b).

Nrg4SD es consciente de que el reto humano, económico y medioambiental que supone el cambio climático no acaba sino de comenzar. Por ello, la red reclama estructuras de gobierno internacionales y nacionales capaces de dar cabida a las acciones de los gobiernos subnacionales y dispuestas a evolucionar hacia una aproximación multidimensional y complementaria en la lucha contra el calentamiento global.

Por último, nrg4SD defiende que la clave para comprender la globalización pasa necesariamente por la creación de una gobernanza moderna y adecuada, capaz de aunar y coordinar los esfuerzos de todos los niveles de gobierno y agentes involucrados. En este sentido, el ingente reto del cambio climático debería entenderse en términos de oportunidad. De hecho, es precisamente en la lectura positiva de los retos del siglo XXI donde surge el liderazgo ambicioso y realista de los gobiernos regionales en la acción climática. 


\section{La Participación de las Comunidades Autónomas Españolas y de los Esta- dos Brasileños en nrg4SD: El Contexto Continental}

$\mathrm{Al}$ observar el listado ${ }^{7}$ de regiones miembro de nrg4SD, es forzoso asentir que las regiones europeas siguen teniendo un mayor peso respecto al resto de los miembros de la red (HAPPAERTS, 2008); y dentro de este mayor número de regiones europeas, son las Comunidades Autónomas (CCAA) españolas las que cuentan con una mayor representación y participación: Aragón, Cataluña y País Vasco y, en su día, Galicia, Cantabria, Islas Canarias y Andalucía. Y por su parte, entre los miembros del hemisferio sur, los 26 estados federados brasileños, sea individualmente, sea vía Asociación de Estados, constituyen la más expresiva representación de un mismo país o de un continente.

El hecho de que las regiones europeas individualmente aun tengan mayor presencia ${ }^{8}$ en nrg4SD que las regiones de otros continentes, tiene su explicación en la existencia de un alto nivel de actividad regional en Europa (JONES y KEATING, 1996; PETSCHEN, 1993; PINTARIS, 1995; DE CASTRO RUANO, 1994; HOOGHE, 1996) la cual se debe principalmente a dos motivos. Por un lado, Europa como comunidad de culturas (MANGAS MARTÍN y LIÑÁN NOGUERAS, 1996; KEATING, 2005) comparte unas raíces históricas, culturales y jurídicas que favorecen la cooperación y compatibilidad de sus regiones. Por otro lado, la Unión Europea (UE), como organización supranacional de integración europea, ha creado amplias oportunidades para que las regiones ejerzan influencia de forma independiente de sus Estados (KEATING, 2005).

A consecuencia de ello, la actividad internacional de las regiones de Europa y concretamente de la UE, se ha plasmado en la participación activa en el Comité de las Regiones, el establecimiento de Oficinas Regionales en Bruselas, la solicitud masiva de proyectos para la cooperación regional con fondos de financiación europea-INTERREG, la cooperación transfronteriza y bilateral en ámbitos específicos y la participación en organizaciones de regiones con objetivos concretos, como la Conferencia de Regiones Marítimas Periféricas-CRPM o, en el ámbito medioambiental, la Conferencia Medioambiental de Regiones de Europa -ENCORE (REI et al., 2013b).

Respecto a las experiencias de desarrollo de actividades paradiplomáticas en América Latina, la tarea ha sido más compleja sobre todo en Estados unitarios con una tradición

$7 \quad$ Vea www.nrg4sd.org/members

8 Hoy en día, las regiones de Latino América y Caribe, principalmente por intermedio de las Asociaciones Regionales de Gobiernos Subnacionales (ABEMA - Asociación de las Entidades Medioambientales Regionales, ANAAE - Asociación Nacional de Autoridades Ambientales Estatales de México, CONAGO - Conferencia Nacional de Gobernadores de México, CONCOPE - Consorcio de Consejos Provinciales de Ecuador, Congreso de Intendentes de Uruguay y OLAGI - Organización Latinoamericana de Gobiernos Intermedios) representan el grupo con mayor presencia en nrg4SD. 
cultural centralista y un régimen político presidencialista. En efecto, todavía la cultura política latinoamericana, y particularmente la diplomática, padece de un marcado hermetismo que hace con que poderes ejecutivos centrales tengan "exclusividad" en la conducción de la acción exterior de los intereses nacionales. Sin embargo, aunque la recién e importantísima proliferación de actores paradiplomáticos no se corresponde con la realidad histórica de la paradiplomacia en Europa, hay que señalar que la toma de decisiones en materia de relaciones exteriores, tradicionalmente en manos de muy pocas personas, sobre todo en regímenes políticos de corte presidencialistas y unitario, va desapareciendo, y de hecho así está sucediendo cada día más, en un entramado cada vez más amplio, complejo y participativo de consejos consultivos, conferencias sectoriales, comisiones asesoras y comités intersectoriales (TORRIJOS, 2000).

En Brasil, algunos estados federados comenzaron a lanzarse directamente en el ambiente internacional, especialmente para buscar alternativas a los problemas de la escasez económica de los años 80, en un escenario de endeudamiento interno, empeorado por la crisis fiscal en la década de 1990. Por otro lado, la globalización y el "Estado logística “ creaba no sólo una oportunidad, sino también una necesidad de la participación de las autoridades regional en la política de comercio exterior (SARAIVA, 2006).

A escala regional, los procesos de integración en el cono sur del hemisferio, han sido poderosos espacios de acción subnacional, ya sea en su dimensión primaria de frontera, ya sea en su vocación progresista de involucrar a los diferentes actores subnacionales en este proceso. En este sentido, la instalación del Foro Consultivo de Municipios, Estados, Provincias y Departamentos del Mercosur en la Cumbre del Mercosur en 2007, ha sido un avance sin precedentes en el reconocimiento de la legitimidad de los gobiernos subnacionales de Brasil para formular y expresar una opinión sobre la política exterior de la proceso de integración (RODRIGUES y KLEIMAN, 2007).

En la última década, en el ámbito ambiental, se registró una cantidad creciente de acuerdos de cooperación técnica entre los estados brasileños (y los municipios) y las contrapartes gubernamentales extranjeras para poner en práctica políticas de protección del medio ambiente y de sostenibilidad teniendo una base de tratados o instrumentos internacionales (RODRIGUES, 2008).

\subsection{Las Actividades Paradiplomáticas Medioambientales de las CCAA y de Ios Estados Brasileños}

Con especial referencia a los ámbitos del medio ambiente y del desarrollo sostenible, se puede afirmar que las CCAA y los estados federados brasileños han encontrado la motivación para desarrollar actividades paradiplomáticas en diversas razones, algunas en común. En primer lugar, las CCAA y los estados brasileños tienen una gran importancia 
espacial y territorial y unas competencias atribuidas por la Constitución Española de 1978 y por la Constitución Brasileña de 1988 que afectan a los ecosistemas, los recursos y la gestión ambiental en general. En segundo lugar, las CCAA son responsables de la implementación de las dos terceras partes de las Directivas europeas en materia medioambiental y en el caso brasileño los estados federados posen competencia legislativa en la materia, inclusive con poder judiciario propio. Es decir, ambos, CCAA y estados brasileños, disponen de importantes competencias en materia de desarrollo sostenible. Y en tercer lugar, son la entidad más cercana al territorio y a los ciudadanos, por lo que están situados en una posición privilegiada para conocer los posibles problemas medioambientales existentes e implementar las necesarias soluciones.

Por estos motivos, así como por la conciencia de que los problemas medioambientales en un mundo globalizado exigen la toma de soluciones más allá de las propias fronteras, las CCAA españolas y los estados federados brasileños han buscado un papel activo, pero diferenciado, en el debate internacional de los asuntos medioambientales -como el cambio climático- con el objeto de que la "voz de las regiones" sea también escuchada en los foros internacionales creados inicialmente para los Estados en exclusiva. En este sentido, Yvo de Boer, ex-Secretario Ejecutivo de UNFCCC afirmó en noviembre de 2010 (en prensa) que la participación de los gobiernos subnacionales en el proceso post-Kyoto era necesaria para el éxito de las negociaciones, teniendo en cuenta que la información bottom-up de la que cuentan los gobiernos subnacionales es imprescindible para la lucha contra el cambio climático (REI et al., 2013b).

Consideramos la participación de las CCAA y de los estados brasileños en la red nrg4SD como un modelo de paradiplomacia regional multilateral de amplio alcance. Para tal consideración, nos basamos en la mayor amplitud y activismo que ofrecen los actores participantes en nrg4SD, lo cual brinda como resultado una agenda internacional multilateral más dinámica y pragmática.

\subsection{Las CCAA Españolas y los Estados Brasileños en nrg4sd}

En la actualidad son miembros de derecho de nrg4SD las siguientes CCAA españolas y los siguientes estados federados brasileños:

1- Aragón, Cataluña, Islas Canarias y País Vasco. La Fundación Comunidad Valenciana-Región Europa figura como miembro asociado. Históricamente, otras CCAA, como Galicia, Cantabria y Andalucía, han formado parte de nrg4SD, aunque en la actualidad ya no ostentan la calidad de miembro.

2- São Paulo, Rio de Janeiro, Goiás y Paraná. Los demás estados brasileños se hacen representados por ABEMA - Associação Brasileira de Entidades de Meio 
Ambiente, aunque en su momento Bahía, Mato Grosso, Paraíba, Pernambuco, Rio Grande do Sul y Tocantins han tenido participación individual, pero no como miembros.

Se puede afirmar que en los casi 15 años de singladura de nrg4SD las CCAA españolas - bajo el liderazgo indudable de Cataluña y País Vasco - y los estados brasileños - bajo el liderazgo evidente de São Paulo - han desarrollado un papel destacado en la vida y gestión de la red. La participación de las CCAA y de los estados brasileños en nrg4SD comienza en el mismo origen de la red, siendo Cataluña. País Vasco, por el lado español, y Goiás, Mato Grosso, Pernambuco, Rio de Janeiro, Rio Grande do Sul y Tocantins, por el lado brasileño, firmantes de la Declaración de Gauteng junto con otros 15 gobiernos subnacionales y 4 asociaciones regionales de gobiernos subnacionales (DECLARACIÓN DE GAUTENG, 2002). Cabe destacar que fueran los estados brasileños la representación numéricamente más significativa de la Declaración. Desde entonces, la participación de las CCAA españolas y de los estados brasileños en los órganos de gestión de la red - Secretariado, Steering Commitee y Co-Presidencia Norte - así como en las actividades programadas por la misma (side-events, cumbres etc.) ha sido una constante y un referente.

\subsection{La Cooperación Hispano-Americana: la Participación Destacada de dos CCAA y de un Estado Brasileño en la Red nrg4sd: País Vasco, Cataluña y São Paulo}

Instituciones como nrg4SD dan buena cuenta de los beneficios que debería reportar la cooperación multilateral multinivel, poniendo de manifiesto lo enormemente positivo que es la práctica del diálogo técnico y del debate sobre las diferencias en el proceso democrático, en el marco de una agenda internacional complexa en el que nos movemos. En otros lugares del mundo, regiones, provincias, departamentos miembros de nrg4SD están experimentando un crecimiento económico sin precedentes. Y eso ha permitido el establecimiento de una vía de diálogo que se plantea a continuación: sobre la base de ese crecimiento económico, no siempre sostenido, hay que crear sistemas políticos justos, sociedades abiertas, inclusivas y respetuosas con los derechos humanos y el medio ambiente.

En estas cuestiones es donde la experiencia de la joven democracia de las autonomías españolas supuso un paso por delante, especialmente en el dialogo inicial con la realidad latinoamericana ${ }^{9}$.

9 La primera Conferencia de América Latina y el Caribe de la Red se celebró en Toluca y Valle de Bravo, en el Estado de México, del 28 al 31 de octubre de 2003 y contó con asistencia vasca, como único miembro europeo presente en la misma. En 2004 se ha celebrado la segunda Conferencia geográfica de la nrg4SD para América y el Caribe en Recife, Pernambuco, del 27 al 28 de agosto, con representación ampliada. En esta Conferencia se firmó un Protocolo de intenciones con la Oficina Regional para América Latina y 
Con el subcontinente americano la vecindad es de otra naturaleza: la proximidad no es física sino que se basa en unos valores y en una visión compartida. En un mundo complejo y en cambio constante, la acción coordinada entre gobiernos de CCAA y sus homólogos argentinos, brasileños, peruanos y ecuatorianos solo pueden y solo deben progresar. Además, cualquier observador imparcial reconoce que América Latina es uno de los grandes beneficiarios del siglo XXI, por lo que una mayor profundización en la relación entre gobiernos regionales de ambos continentes constituye un elemento clave para el desarrollo de la actividad paradiplomatica.

El País Vasco ha sido uno de los miembros más comprometidos con nrg4SD y uno de sus mayores valedores. Como se ha mencionado más arriba, el País Vasco fue una de las regiones involucradas en el origen de nrg4SD, y su compromiso quedó pronto patente al albergar la Conferencia de marzo de 2003, en la que los gobiernos subnacionales firmantes de la Declaración de Gauteng se reunieron para desarrollar la idea que germinó en Johannesburgo. Concretamente, en la Conferencia de marzo de 2003 se acordaron la estructura inicial de la red así como su denominación: red de gobiernos subnacionales por el desarrollo sostenible - nrg4SD (LOPERENA ROTA y EZEIZABARRENA SÁENZ, 2004). En esta estructura inicial de nrg4SD (en la que se concibió un Secretariado, un Steering Commitee, y las Co-Presidencias Norte y Sur) cabe destacar la asunción de responsabilidades del País Vasco como reflejo del gran interés y compromiso de esta Comunidad Autónoma (CA) con el proyecto. Efectivamente, el País Vasco solicitó un puesto en el Steering Commitee de la red - el cual ha mantenido con las oportunas renovaciones desde 2003 hasta la actualidad - y ostentó la Co-Presidencia Norte en el período 20052008. Además, el País Vasco asumió generosamente - sin cargo financiero para la red - la gestión del Secretariado de la red de forma provisional (en la práctica fue desempeñado hasta junio de 2009) hasta que éste se profesionalizara (REI et al., 2013b).

A nivel de representación política, cabe destacar la regularidad de la presencia al más alto nivel del gobierno del País Vasco - Consejera, vice consejero - en las reuniones de la red, lo cual ha permitido mantener su participación viva y enérgica a lo largo de estos años.

En el ámbito técnico, gracias al País Vasco se han logrado algunos policy papers, determinados estudios - como el desarrollo de indicadores subnacionales para el desarrollo sostenible -, y el Memorando de Entendimiento en materia de agua entre las CCAA españolas y las regiones de Latinoamérica y Caribe. Éste último es un mecanismo flexible diseñado como un marco general en el que pueden crearse iniciativas bilaterales que introducen las diversas realidades regionales y amplían los márgenes técnicos de cooperación.

el Caribe del Programa de Naciones Unidas para el Medio Ambiente, por el que se establece un programa marco de cooperación multilateral a favor del desarrollo sostenible al nível regional para América Latina y Caribe. 
Los motivos del País Vasco para formar parte de nrg4SD son funcionales - el desarrollo de las competencias asumidas -, y claramente identitarios. Esto último se deriva de su personalidad histórica, que le lleva a buscar en nrg4SD una nueva forma de relacionarse con otros actores internacionales así como una plataforma para estar presente en la escena internacional - tanto a nivel europeo como mundial - mediante una "ruta extra-estatal" (HAPPAERTS, 2008).

Para rematar, debe afirmarse que el gran protagonismo que el País Vasco ha tenido en los primeros dos tercios de vida de nrg4SD (durante los que llegó a ostentar simultáneamente la Co-Presidencia Norte, la Secretaría y un puesto en el Steering Commitee) han influido muy significativamente en el desarrollo de la red, de forma que podemos afirmar que el País Vasco es uno de los grandes artífices de la configuración actual y trayectoria de nrg4SD.

Cataluña es otra CA con un gran peso histórico en nrg4SD y gran influencia en la evolución de la red. Miembro de la red desde la Declaración de Gauteng, ha tenido un puesto en el Steering Commitee desde los inicios, el cual ha mantenido en los oportunos procesos de renovación de mandato. Asimismo, Cataluña ostenta en la actualidad la Co -Presidencia Norte de la red, habiendo compartido la primera mitad del mandato 20102013 con Aragón. La doble candidatura de Aragón y Cataluña, así como su apoyo mutuo en el mandato tuvo su razón en las elecciones previstas en Cataluña en noviembre de 2010 y en Aragón en mayo de 2011, situación que motivaba una unión de fuerzas para poder cumplir con los compromisos adquiridos para con la red en lo que a la postre fueron sendos cambios de gobierno autonómicos. La Co-Presidencia Norte ejercida por Cataluña es no solo un cargo justo desde el punto de vista de las capacidades de la CA, sino también un merecido reconocimiento del compromiso y entrega constantes de Cataluña hacia nrg4SD a lo largo de sus 10 años de vida (REI et al., 2013b).

A nivel técnico, deben destacarse las valiosas aportaciones catalanas al grupo de trabajo de nrg4SD sobre negociaciones en cambio climático. Desde este grupo, y gracias al trabajo de la CA se presentaron enmiendas al texto de las negociaciones de la Cumbre de Poznan en 2009, momento desde el cual se siguieron las negociaciones para que el elemento subnacional siguiera vivo en el texto, hasta que en 2010 (en Cancún) se hizo un reconocimiento del papel de los gobiernos subnacionales por el $A W G-L C A$ (The Ad Hoc Working Group on Long-term Cooperative Action under the Convention) bajo UNFCCC.

Se puede afirmar que la participación de Cataluña en nrg4SD se debe a un doble objetivo. Por un lado, Cataluña ha querido desarrollar un objetivo funcional - ejercer las competencias medioambientales asumidas constitucionalmente - y por otro lado ha buscado una plataforma hacia el exterior en la que poder expresar sus reivindicaciones identitarias, lingüísticas y culturales. Esto último se ha desarrollado asumiendo y respetando la 
competencia exclusiva de la Administración central en materia de relaciones internacionales, lo cual se aprecia, por ejemplo, en la acreditación del consejero de medio ambiente y ordenación territorial catalán bajo la delegación española en las COP (REI et al., 2013b).

Por último, debe mencionarse que el compromiso de Cataluña con nrg4SD también ha quedado patente en su presencia regular y participación activa en todas las reuniones de la red (tanto de los órganos gestores como de eventos y Cumbres) a un alto nivel político desde la creación de nrg4SD.

São Paulo, por su parte, se incorporó a nrg4SD en la Conferencia de marzo de 2003 cuando se acordaron la estructura inicial de la red. De hecho, São Paulo fue invitado por una iniciativa del País Vasco a asumir la Vice-Presidencia para América Latina y el Caribe (IRIARTE IRURETA, 2006), bien como un puesto en el Steering Commitee de la red el cual ha mantenido con las oportunas renovaciones desde 2003 hasta la actualidad - y ostentó la Co-Presidencia Sur en los períodos 2007-2010 y 2011-2014.

A nivel de representación política, cabe destacar la regularidad de la presencia al más alto nivel del gobierno de São Paulo - Secretario de Estado y/o Presidente de la Agencia Ambiental - en las reuniones de la red, lo cual ha permitido mantener su participación viva y comprometida a lo largo de estos ańos (REI et al.,2013).

En el ámbito técnico, gracias a São Paulo se han logrado algunos policy papers, determinados estudios y entrenamientos - inventario de gases de efecto invernadero para regiones latinoamericanas -, y una asistencia técnica a las regiones de Latinoamérica y Caribe, especialmente facilitada por OLAGI - Organización Latinoamericana de Gobiernos Intermedios. Éste último en un ambiente político de acercamiento en el que se han creado iniciativas bilaterales de cooperación técnica, muchas en cooperación con el País Vasco y/o Cataluña.

Los motivos de São Paulo para formar parte de nrg4SD son igualmente funcionales -el desarrollo de las competencias asumidas -, y reservadamente identitarios. Esto último se deriva de su relevancia económica y cultural en la sociedad brasileña, además de las incongruencias (FARIAS y REI, 2013) de las acciones del país en el régimen internacional de los cambios climáticos, que le lleva a buscar en nrg4SD una forma de relacionarse con otros actores internacionales comprometidos con una agenda global, así como una plataforma para estar presente y actuante en la escena internacional - tanto a nivel latinoamericano como mundial - mediante una "acción verdaderamente exterior, pero disfrazada".

Por último debe afirmarse que el protagonismo que Sáo Paulo ha tenido en la construcción de nrg4SD han influido muy significativamente en el desarrollo de la red, en particular en su expansión latinoamericana, de forma que podemos afirmar que São Paulo junto al País Vasco y Cataluńa son, además de los grandes responsables por el diseño estratégico e implantación de una cooperación descentralizada ibero-americana, eje 
central de la relación norte-sur de nrg4SD, junto a otros miembros artífices principales de la configuración actual y trayectoria de nrg4SD.

Por otro lado, no se puede ignorar la larga tradición europeísta de las CCAA históricas, ya sea del nacionalismo catalán o del vasco (BARBERÁ y BARRIO, 2006), y el histórico autonómico de São Paulo (DOLHNIKOFF, 2005; HOLANDA, 1985) han significado un gran esfuerzo y compromiso para incluir en la agenda internacional del desarrollo sostenible (y en particular en la de lucha contra el cambio climático), un nuevo concepto de soberanía frente al tradicional del Estado central. Todo ello ha contribuido a reforzar la autonomía de esas regiones y a aumentar su voz en el mundo.

Finalmente, es indudable la aportación cuantitativa que las CCAA españolas y los estados brasileños realizan a nrg4SD con su compromiso y trabajo, pero cabe destacar además su contribución cualitativa a la red, la cual ha permitido el estrechamiento de las relaciones de España con las regiones de Latino América y Caribe - con quienes comparten unas importantes raíces lingüísticas y culturales - fruto del cual se han desarrollado destacables colaboraciones. Es importante señalar que este estrechamiento en su día intentó abarcar a Portugal. Sin embargo, en virtud de ser Portugal un Estado unitario, el interés de los archipiélagos de Madeira y Azores en hacerse miembros de nrg4SD se limitó a participaciones puntuales en las Asambleas Generales de la red.

En este sentido, se puede afirmar que la paradiplomacia ambiental del siglo XXI en Latinoamérica pasa necesariamente por las acciones institucionales de nrg4SD en la región ${ }^{10}$ y por las acciones concertadas con las CCAA espańolas y los estados brasileños. Efectivamente, la colaboración de las regiones de Latino América y Caribe, y las CCAA representan en la actualidad un eje propio - con vigor y liderazgo - de actividades en el régimen multilateral de nrg4SD. En este sentido, la última Presidencia de nrg4SD no fue sino el reflejo de este estrechamiento: la Co-Presidencia Norte está en Cataluña, que se mantiene, y la Co-Presidencia Sur en São Paulo.

\section{Conclusiones}

Es indiscutible que lo reto humano, económico y ambiental que residen en la agenda global de la sostenibilidad, exige la existencia de estructuras de gobiernos internacionales

10 El Gobierno del Estado de México recibió la Primera Conferencia Continental de la Red, en Toluca, del 28 al 31 de octubre de 2003. Menos de un año después, del 27 al 28 de agosto de 2004, en Recife, ABEMA promueve la Segunda Conferencia Continental, que centró su labor en el establecimiento de diagnósticos ambientales y las estrategias regionales para el desarrollo sostenible, la estructuración de la Agenda 21 local, en acciones concretas hacer frente al cambio climático y el aumento del uso de energías renovables por los gobiernos de la red. La Tercera Conferencia, en marzo de 2008, se celebra en Sao Paulo, bajo el tema del cambio climático. La cuarta y última edición, celebrada en Mar del Plata en noviembre de 2010 cuidó del tema del agua y comenzó los primeros trabajos derivados del Memorando Multilateral de Entendimiento sobre el Agua para América Latina y el Caribe y la Península Ibérica, firmado también en 2010 en la Tercera Cumbre Mundial de Regiones en Montevideo. 
y nacionales capaces de reflejar el papel crucial que los gobiernos subnacionales desempeñan en este desafío global. En este sentido, la paradiplomacia ambiental supone una aproximación multidimensional, complementaria y coherente en esa agenda, en particular en la lucha contra el cambio climático. Efectivamente, la interpretación positiva de los retos del siglo XXI permite la transición hacia una gobernanza moderna y correcta, donde haya cabida para el liderazgo realista y ambicioso de los gobiernos subnacionales en la acción climática.

Durante sus casi 15 años de existencia, nrg4SD ha trabajado para crear un escenario internacional en el que haya cabida e espacio para las acciones y voces subnacionales. Ello ha contribuido a la creación de un movimiento legal innovador, cuya base es un trabajo normativo que debe ser actualizado y revisado de forma permanente. Y en la creación de ese movimiento, consideramos la participación de las CCAA y de los estados federados brasileńos en la red nrg4SD como un modelo de paradiplomacia regional multilateral de amplio alcance y de refinada estrategia.

A pesar de las diferencias en las estrategias, los vínculos o las cuestiones de identidad nacionalista o autonómica de las regiones en su proyección hacia el exterior, la experiencia paradiplomática hispano-brasileña ha funcionado como ejemplo de unión de fuerzas y apoyo mutuo para reclamar en el seno de foros internacionales (y particularmente en el Sistema de NNUU) la importancia del papel de los gobiernos subnacionales y sus redes, tanto en la gestión de asuntos multilaterales de desarrollo sostenible, como en la identificación práctica de oportunidades de cooperación específica entre regiones del mundo.

\section{Referencias}

ALDECOA, F., KEATING, M. Paradiplomacia: Las relaciones internacionales de las regiones. Marcial Pons. Madrid: Barcelona, 2010.

BARBERA, O., BARRIO,A. Barrio. Convergencia i Unió: from Stability to decline. In: WINTER, L, GOMEZ-REINO, M. y LYNCH, P. (eds.) Autonomist Parties in Europe: Identity Politics and the Revival of the Territorial Cleavage. Barcelona: ICPS, 2006.

BERSTEIN, S., BETSILL, M., HOFFMANN, M., PATERSON, M. A tale of two Copenhagens: carbon markets and climate governance. Millennium - Journal of International Studies, vol. 39, n. 1, p.161-173, 2010.

BUENO DA SILVA DA SILVA, E. Contemporary paradiplomacy: trajectories and trends of international actions by state governments of Brazil and the US. Doctorate thesis submitted to the International Relations Doctorate Program of the International Relations Institute of the University of Brasília (IREL/UnB) for obtaining a doctor degree in international relations, 2010. 
BULKELEY, H. Reconfiguring environmental governance: towards a politics of scales and networks. Political Geography, n. 24, p.875-902, 2005.

. Cities and the Governing of Climate Change. Annual Review of Environment and Resources, 35(1), p. 229-253, 2010.

BULKELEY, H. and NEWELL, P. Governing Climate Change. London and New York: Routledge, 2010.

DE CASTRO RUANO, J.L., La emergente participación política de las regiones en el proceso de construcción europea. Vitoria: Instituto Vasco de Administración Pública, 1994.

DECLARACIÒN DE GAUTENG. Disponible en http://www.palencia21rural.com/ doc/declaracion_gauteng.pdf. Aceso en 20 de abril de 2015.

DEDUERWAERDERE, T. The contribution of network governance to sustainable development. Belgium: Université Catholique de Louvain Fonds National de la Recherche Scientifique, feb. 2005.

DOLHNIKOFF, M. O pacto imperial: origens do federalismo no Brasil. São Paulo: Globo, 2005.

FARIAS, V.C., REI, F. O regime jurídico nacional de combate às mudanças climáticas e suas incongruências. In GRANZIERA,M.L.M., REI, F. Petróleo, gás e meio ambiente. Santos: Editora Universitária Leopoldianum, 2013, p.133-158.

FOGAR. www.regionsunies-fogar.org/es/. Aceso en 2 de abril de 2015.

GRAZIANO, X., REI, F. Mudanças climáticas e São Paulo. Folha de São Paulo, São Paulo, edición 17/02/2009, p. A3, 2009.

HAPPAERTS, S., VAN DEN BRANDE, K., BRUYNINCKX, H. Governance for sustainable development at the inter-subnational level: The case of the Network of Regional Governments for Sustainable Development (nrg4SD), Working paper $\mathrm{n}^{\circ} 3$, February 2008.

HOCKING, B. Regionalismo: uma perspectiva das relaçôes internacionais. In: VIGEVANI, T., WANDERLEY, L.E., BARRETO, M.I., MARIANO, M.P (org.). A dimensão subnacional e as relaçóes internacionais. São Paulo: EDUC; Fundação Editora da UNESP; Bauru: EDUSC, 2004, p.77-108.

HOLANDA, S.B. "São Paulo”. In: S. B. de Holanda (org.) - História Geral da Civilizaçáo Brasileira. 5a. ed., São Paulo: Difel, T.II, 2o. V, 1985.

HOOGHE, L. Cohesión Policy and European Integration, Oxford: Claredon, 1996.

IRIARTE IRURETA, I. La colaboración entre Gobiernos Regionales: la Red internacional de Gobiernos Regionales por el Desarrollo Sostenible (nrg4SD). In XVI Congre- 
so de Estudios Vascos: Garapen Iraunkorra. Donostia: Eusko Ikaskuntza, 2006, p. 531-533.

JONES B., KEATING M. The European Union and the Regions, Oxford: Clarendon, 1996.

KEATING, M. Regions and international affairs: motives, opportunities and strategies. In: ALDECOA, F. KEATING, M. Paradiplomacy in action: the foreign relations of subnational governments. London: Frank Cass Publishers, 2005, p.875-902.

LITFIN, K.T. Environment, Wealth and Authority: global climate change and emerging modes of legitimation. International Studies Review, vol.2, n. 2, 2000, p.119-148.

LOPERENA ROTA D. AND EZEIZABARRENA SÁENZ X. Network of Regional Governments for Sustainable Development. Revista Medi ambient, no. 35, 2004.

LUNA PONT, M., Las relaciones internacionales subnacionales desde la teoría de las relaciones internacionales. Revista TIP, año1, no1, 2011, p. 64-83.

MANGAS MARTÍN, A., LIÑÁN NOGUERAS D.J, Instituciones y Derecho de la Unión Europea. Madrid: Tecnos, 1996.

MATEO, R.M. Tratado de derecho ambiental. Vol II. Madrid: Trivium, 1992.

MILANI, C.R.S., RIBEIRO, M.C.M. International relations and the paradiplomacy of Brazilian cities: crafting the concept of local international management. Brazilian Administration Review, vol. 8., n. 1, jan-mar 2011.

nrg4SD. Network of Regional Governments for Sustainable Development. Disponible en http://www.nrg4sd.org/. Aceso en 2 de abril de 2015.

nrg4SD. The Cancun Agreements - Overview of the main outcomes of UNFCCC COP 16 / CMP 6. Nrg4SD, 2011.

OECD. Governance for Sustainable Development. Five OECD case studies, OECD Publications, Paris: OECD, 2002.

OKEREKE, C. BULKELEY, H. SCHROEDER, H. Conceptualizing climate governance beyond the international regime. Global Environmental Politics, vol.9, n.1, 2009, p. 58-68.

PETSCHEN S. La Europa de las Regiones, Barcelona: Generalitat de Catalunya, 1993.

PINTARIS S. Macht, Demokratie und Regionem in Europa. Analysen und Szenarien der Integration und Desintegration. Marburg: Metropolis, 1995.

RABE, B. G. Statehouse \& Greenhouse: The Emerging Politics of American Climate Change Policy. Washington, DC: Brookings Institution Press, 2004. 
REI, F.C.F., CUNHA, K.B., SETZER, J. La Paradiplocia Ambiental en la Nueva Governanza Internacional. Revista TIP n.2. Buenos Aires, 2012, p. 50-63.

Paradiplomacia Ambiental: a participaçáo brasileira no regime internacional de mudanças climáticas, Revista de Direito Ambiental, v. 71, 2013, p.265-286.

REI, F.; CUNHA, K.; VERA, N. La paradiplomacia medioambiental global y el papel de las comunidades autónomas españolas. Revista Foro Internacional, v. LIII 2, n. 212, Mexico, 2013b, p. 337-362.

RODRIGUES, G.., KLEIMAN, A. The MERCOSUR Committee of Municipalities, States, Provinces and Departaments. Process of Creation and Perspectives. Fifth Annual Conference of the Euro-Latin Study Network on Integration and Trade ELSNIT, Barcelona, 26 e 27 de octubre 2007.

RODRIGUES, G. Relaçóes internacionais federativas no Brasil. Dados, v. 51, n.4, Rio de Janeiro, 2008.

ROSENZWEIG, C., SOLECKI, W., HAMMER, S. A. \& MEHROTRA, S. Cities lead the way in climate-change action. Nature 467, 2010, p. 909-911.

SETZER, J. Governança multinível das mudanças climáticas: políticas subnacionais e ações transnacionais em São Paulo. In: REI, F.C.F. (org.). Direito e desenvolvimento: uma abordagem sustentável. São Paulo: Saraiva, 2013.

SARAIVA, J.F.S. Federalismo e Relações Internacionais do Brasil. In: ALTEMANI, H. e LESSA, A. C. (orgs.). Relaçóes Internacionais do Brasil. Temas e agendas. São Paulo: Saraiva, vol. 2, 2006, p. 429-455.

TORRIJOS, Vicente, “La diplomacia centrífuga. Preámbulo a una política exterior de las regiones”, Desafíos, no 2, 2000, p. 19-54.

UNFCCC. Report of the Conference of the Parties on its sixteenth session, held in Cancun from 29 November to 10 December 2010. UNFCCC, March 2011. 